\title{
Heat and mass transfer in a liquid film flowing down the bottom side of inclined surface
}

\author{
Maria V. Bartashevich ${ }^{1,}$, Maxim G. Vlasenko ${ }^{1}$, Andrey A. Pil'nik ${ }^{1}$, and Andrey A. Chernov ${ }^{1}$ \\ ${ }^{1}$ Kutateladze Institute of Thermophysics, 630090 Novosibirsk, Russia
}

\begin{abstract}
The work presents derivation of the equation describing the shape of the free surface of a liquid film flowing along the bottom wall of an inclined surface, taking into account conjugate heat and mass transfer under the condition that the temperature and concentration on the free surface are unknown in advance and interrelated.
\end{abstract}

\section{Problem statement}

This work deals with investigation of combined heat and mass transfer on a thin film of liquid (water solution) moving along the bottom side of an inclined surface. The similar problems were considered in [1-6]. So, the analytical solutions to the simplest problems on combined heat and mass transfer at absorption on free surfaces of various geometries are presented in [1]. Heat and mass transfer in the entrance region of the falling axisymmetric semi-infinite film surrounded by the pure vapour was studied in [2]. A stationary axisymmetric flow of a thin liquid film along a confuser is considered in [3], the rivulet flow on the bottom wall of an inclined cylinder is analyzed in [4]. The exact solution to the problem of isothermal diffusion to a wave film was obtained in [5]. The mathematical model of combined heat and mass transfer on a vaporizing laminar liquid film of a constant thickness, flowing along a vertical cylinder, is presented in [6]. To solve the heat and mass transfer equations, Laplace transformations were used, and comparisons with experimental data were made. In this paper, we present an equation describing the shape of the free surface of a liquid film flowing down the bottom side of an inclined surface with consideration of conjugate heat and mass transfer.

We consider the two-dimensional stationary flow of a laminar liquid film of thickness $h$ over a plate inclined at angle $\alpha$ to the horizon, as schematically shown in Fig. 1. The surface of liquid is in contact with immobile vapor. The temperature and concentration on the free surface are not known in advance and interrelated. The thermal-physical properties of solution are assumed constant.

\footnotetext{
* Corresponding author: bartashevichmv@gmail.com
} 


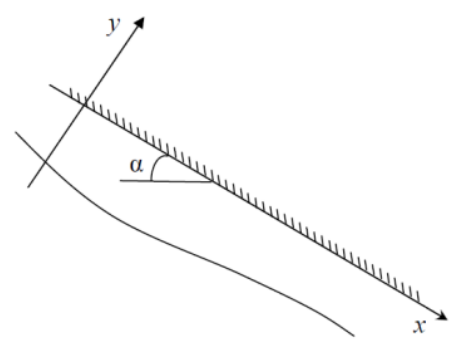

Fig. 1. Scheme of the problem of heat and mass transfer in a liquid film flowing down the bottom side of inclined surface.

\section{Film thickness equation}

Let us introduce the Cartesian coordinate system with $O x$ axis in the direction of the flow and $O y$ axis directed along the normal to the plate. It is assumed that in the region of liquid flow the following equations are satisfied:

$$
\begin{gathered}
u \frac{\partial u}{\partial x}+v \frac{\partial u}{\partial y}=-\frac{1}{\rho} \frac{\partial p}{\partial x}+v \frac{\partial^{2} u}{\partial y^{2}}+g \sin \alpha \\
-\frac{1}{\rho} \frac{\partial p}{\partial x}-g \cos \alpha=0 \\
\frac{\partial u}{\partial x}+\frac{\partial v}{\partial y}=0
\end{gathered}
$$

The boundary conditions for systems (1) - (3) take the following form.

At $y=0$

$$
u=v=0 \text {. }
$$

At $y=-h$

$$
\begin{aligned}
& \frac{\partial u}{\partial y}=0 \\
& p=p_{a}-\sigma \frac{\partial^{2} h}{\partial x^{2}} . \\
& u \frac{\partial h}{\partial x}-\frac{\lambda}{\rho r} \frac{\partial T}{\partial y}-\frac{D}{\rho} \frac{\partial C}{\partial y}=v
\end{aligned}
$$

It follows from (2) and (6) that

$$
p=p_{a}+\rho g(h(x)-y) \cos \alpha-\sigma \frac{\partial^{2} h}{\partial x^{2}} .
$$

From (1) and (8) we obtain 


$$
u \frac{\partial u}{\partial x}+v \frac{\partial u}{\partial y}=-g \frac{\partial h}{\partial x} \cos \alpha+\sigma \frac{\partial^{3} h}{\partial x^{3}}+v \frac{\partial^{2} u}{\partial y^{2}}+g \sin \alpha
$$

Integrating (9) with consideration of (3) and (7), we obtain equation

$$
\frac{\partial J}{\partial x}+g h \cos \alpha \frac{\partial h}{\partial x}-v \int_{0}^{h} \frac{\partial^{2} u}{\partial y^{2}} d y-g h \sin \alpha+\frac{\sigma h}{\rho} \frac{\partial^{3} h}{\partial x^{3}}-\frac{u_{i} \lambda}{\rho r} \frac{\partial T}{\partial y}-\frac{u_{i} D}{\rho} \frac{\partial C}{\partial y}=0,
$$

where $J=\int_{0}^{h} u^{2} d y$. Let the velocity profile is

$$
u=-\frac{g h^{2} \sin \alpha}{2 v}\left(\frac{2 y}{h}+\frac{y^{2}}{h^{2}}\right)
$$

We should note that conditions (4) and (5) are satisfied. Then, the surface equation will take form:

$$
\frac{57}{40} \frac{\partial}{\partial x}\left(\frac{q^{2}}{h}\right)+g h \frac{\partial h}{\partial x} \cos \alpha+\frac{3 q v}{h^{2}}+\frac{\sigma h}{\rho} \frac{\partial^{3} h}{\partial x^{3}}-u_{i} \frac{\lambda}{\rho r} \frac{\partial T}{\partial y}-u_{i} \frac{D}{\rho} \frac{\partial C}{\partial y}=0,
$$

where $q=\int_{0}^{h} u d y, u_{i}=\frac{3}{4} \frac{q}{h}$

\section{Conclusions}

The work presents derivation of the equation describing the shape of the free surface of a liquid film flowing along the bottom wall of an inclined surface, taking into account conjugate heat and mass transfer under the condition that the temperature and concentration on the free surface are unknown in advance and interrelated. The numerical solution of the resulting equation is a separate complex and non-trivial problem and it will be the subject of further research of the authors.

The work was supported by the grant MK-6384.2016.8.

\section{References}

1. V.E. Nakoryakov, N.I. Grigorieva, Non-Isothermal Absorption in Thermal Transformers (Nauka, Novosibirsk, 2010)

2. V.E. Nakoryakov, N.I. Grigoryeva, M.V. Bartashevich, Int. J. Heat Mass Transfer. 54, $21(2011)$

3. L. I. Sen', A. M. Te, O. Yu. Tsvelodub, J. Appl. Mech.Tech. Phys. 24, 3 (1983)

4. S.V. Alekseenko, P.I. Geshev, P.A. Kuibin, Doklady Physics, 42 (1997)

5. P.I. Geshev, A.M. Lapin, J. Appl. Mech.Tech. Phys. 24, 6 (1983)

6. Y. Wu, Int. J. Heat Mass Transfer. 93 (2016) 